A HISTORIOGRAPHICAL PERSPECTIVE:

REPUBLICAN ITALY, REPRESENTATION

AND GENDER REPRESENTATIONS (1945-1968)

\title{
Una prospettiva storiografica: l'Italia repubblicana, la rappresentanza, le rappresentazioni di genere (1945-1968)
}

Patrizia Gabrielli

\section{Università di Siena}

patrizia.gabrielli@unisi.it - https://orcid.org/0000-0001-5579-4686

Fecha recepción: 01.07.2019/Fecha aceptación: 06.10.2019

\section{Sinopsi}

La storiografia ha esaminato con originalità e da diverse prospettive il suffragio e la rappresentanza ma ulteriori approfondimenti possono giungere dallo studio dei significati che l' «universo semiotico» della politica attribuisce alle differenze di genere nello spazio pubblico. Sulla base di una ricerca sulle fonti a stampa e d'archivio, il saggio esamina le pratiche discorsive sull' «uomo pubblico» e sulla «donna pubblica» nei primi vent' anni dell' Italia repubblicana. Al fine di mettere in evidenza le tante eredità del passato sulla politica italiana, offre alcu-

\begin{abstract}
Historiography has examined suffrage and representation with originality and from different perspectives, but further elucidation can come from a study of the meanings that the "semiotic universe" of politics attributes to gender differences in public space. Based on research using press and archive sources, this article examines discursive practices in relation to "public man" and "public woman" in the first twenty years of Republican Italy. In order to highlight the many legacies of the past in Italian politics, I offer some insights into representations
\end{abstract}


Miscelánea | Una prospettiva storiografica: l'Italia repubblicana, la rappresentanza, le rappresentazioni di genere (1945-1968)

ni spunti sulle rappresentazioni della femminista di primo Novecento e sulla «sovversiva» negli anni del regime fascista.

\section{Parole chiave}

Italia repubblicana, storiografia, genere, rappresentanza, rappresentazioni. of "feminist" in the early twentieth century and "subversive" during the fascist regime.

Keywords

Italian Republic, historiography, gender, representative, representations. 
LE IMPRESSIONI DEL PRIMO INCONTRO con le deputatesse si possono così riassumere: non fumano, in genere, e in maggioranza non si truccano, e vestono con la più grande semplicità ${ }^{1}$.

\section{Squilibrio di genere nella rappresentanza}

Ad oltre settant'anni dal voto e nonostante i recenti provvedimenti legislativi ${ }^{2}$, lo squilibrio di genere nella rappresentanza è in Italia ancora un dato evidente, al centro del dibattito politico e scientifico.

Per quanto concerne la storiografia, l'attenzione alla dimensione di genere sull' Italia Repubblicana si afferma alla metà degli anni Novanta, nel pieno della fioritura di una articolata bibliografia su quella stagione. A segnare la svolta è il 1996, Cinquantesimo anniversario del diritto di voto alle donne in Italia, con il piccolo ma proficuo volume di Anna Rossi Doria - preceduto due anni prima (nel 1994) da un «robusto» saggio dell'Autrice ${ }^{3}$ - e con un apprezzato articolo di Annarita Buttafuoco ${ }^{4}$. Pur nelle differenze, le due studiose, attente alla dimensione orizzontale e verticale degli eventi, collocando il decreto del 1945 sul suffragio nel lungo periodo, ovvero nella tradizione dei movimenti suffragisti, lo interpretano alla stregua di un evento periodizzante da cui scaturisce la definizione di un modello di cittadinanza che include il genere. A partire dal 1996, dunque, l'evento è stato sottoposto a un vaglio cri-

1. "Le 21 donne alla Costituente", La Domenica del Corriere: supplemento illustrato al Corriere della Sera, 4 agosto $1946,3$.

2. “Parità vo' cercando - 1978-2018. Le donne italiane in settanta anni di elezioni”, Dossier del Senato della Repubblica, 13, 2018: «Nel 1993, con la disciplina della formazione delle liste dei candidati e nel 2001-2003 le pari opportunità sono entrate nella Costituzione. Nel 2015, l'Italicum stabilisce l'obbligo di rappresentanza paritaria dei due sessi nelle candidature circoscrizionali, e nel 2017, con la legge n.165, la promozione femminile è diventata un obbligo dei partiti con l'alternanza di genere nella sequenza della lista, la quota di genere nelle candidature uninominali e la quota di genere nella posizione di capolista per i collegi plurinominali».

3. Si veda A. Rossi-Doria, "Le donne sulla scena politica”, in Storia dell' Italia repubblicana, Torino, 1994, vol. I, La costruzione della democrazia. Dalla caduta del fascismo agli anni cinquanta, 778-846; Ead., Diventare cittadine. Il voto alle donne in Italia, Firenze, 1996.

4. A. Buttafuoco, "Cittadine italiane al voto", in Passato e presente, n. 40, 1997, 5-11. 
tico, si sono messe in luce le ricadute, le ambiguità e le contraddizioni. Non è questa la sede per esaminare nel dettaglio questa produzione ma, molto sinteticamente, si può affermare che al suo interno si possono individuare sostanzialmente due principali filoni. Il primo, focalizzando l'attenzione sulla Costituzione, ha messo in luce la fragilità della posizione lavorativa e professionale delle donne, gli squilibri sul piano dei diritti civili, da cui matura una «disincrasia» tra uguaglianza nella sfera pubblica e inferiorità nella sfera privata. Sempre lungo questo filone di ricerca, altre studiose hanno valorizzato il dibattito alla Costituente, posto l'accento sulle difficoltà, se non gli ostacoli, incontrate dalle Consultrici e sul lavoro di mediazione da esse svolto nella definizione di molti articoli del dettato costituzionale, non ultimi quelli inerenti il valore sociale e le garanzie per la maternità. Meno praticato, invece, il metodo biografico. Sulle madri della Repubblica, se si esclude la memorialistica, si hanno a disposizione voci biografiche ma non biografie compiute, rare, se non addirittura assenti, le ricerche sulle elette alle successive tornate elettorali ${ }^{5}$.

La memorialistica e le testimonianze orali hanno aperto ampie prospettive alla ricerca anche su questi specifici temi. Molte studiose, avvalendosi del ricco e nutrito dibattito sul genere autobiografico, si sono proposte di cogliere il «senso»e il «valore» attribuiti dai soggetti all'esperienza di elettrici e di elette. Si è così disegnata un'articolata geografia dei «vissuti» (di esperienze di donne) che hanno consentito una diversa considerazione del significato attribuito al suffragio dalle italiane, a lungo viste come poco interessate, se non addirittura estranee, a quel diritto. Se sono state in prima battuta le testimonianze delle intellettuali - è il caso di quelle raccolte da Alba de Céspedes nel fascicolo di «Mercurio» del $1946^{6}$ - a dare la misura della forza dirompente per alcune di quell'evento, in un secondo momento le testimonianze delle «donne comuni» hanno permesso di riconsiderare un passaggio certo non marginale nella costruzione di una identità di genere e delle aspettative che l'esercizio di quel diritto apriva.

Se questi sono i temi emersi dal dibattito storiografico, merita sottolineare che cittadinanza e rappresentanza femminile sono state affrontate da molteplici prospettive. Ricerche e studi hanno messo a fuoco i fattori e le variabili che vi concorrono, compresi gli stereotipi di genere, alla cui definizione partecipano la propaganda e i media «luogo di costruzione e di diffusione di rappresentazioni vincolate da apparati ideologico culturali» ${ }^{7}$. Ancora oggi, l' ingresso nelle istituzioni rappresentative di soggetti difformi dal genere maschile produce visioni distorte. Il linguaggio dei loro corpi è distante dalla norma semantica dominante, sintetizzata (e interiorizzata) dall' uomo decoroso, a lungo unico indiscusso titolare di cariche pubbliche. In sintesi, queste reazioni rivelano la rappresentanza non detta del corpo maschile che fa degli «altri»

5. C. Dau Novelli, "Introduzione", en M.T.A. Morelli (Dir.), Le donne della costituente, Roma-Bari, 2007, V-XXVIII; P. Gabrielli, Il primo voto. Elettrici ed elette, Roma, 2016. Entrambi i volumi pubblicano brevi profili sulle costituenti.

6. Mercurio. Processo al '46, III, n. 27-28, 1946.

7. B. M. Mazzara, I discorsi dei media e la psicologia sociale. Ambiti e strumenti d'indagine, Roma, 2008. 
soggetti degli «invasori dello spazio» ${ }^{8}$. Nel 2006 con il governo Berlusconi, nel 2008 con quello Monti, non diversamente nel 2014 con il governo Renzi, il look delle ministre è attentamente vagliato e soppesato dalla stampa intenta ad elargire giudizi su tacchi (sempre troppo alti o troppo bassi), su abiti e accessori elevati a veri e propri metri di misura, utilizzati a favore o contro ministre e parlamentari. Merita sottolineare con Elisa Giomi che, lo stile, qualunque sia, è al centro di critiche che producono effetti normativi. Nessun manifesto interesse, invece, per cravatte e calzature indossate dai leader politici (con l'eccezione di Berlusconi), incolumi dalla «norma dell'esposizione» ${ }^{9}$. Pur mutando nel tempo, tali raffigurazioni accompagnano l'accesso delle donne alla politica e presentano analogie di fondo evidenti nel lungo periodo, quali, ad esempio, la retorica del ridicolo con i suoi effetti svalutativi. Il tema trova espressione negli abbondanti riferimenti alla vacuità che è antitetica alla compostezza, al rigore, alla razionalità, qualità questa, che il governo della cosa pubblica richiede, accuratamente disposte nel bagaglio dell" «uomo politico», espressione della borghesia trionfante, accuratamente precisato tra Otto e Novecento ${ }^{10}$. L'argomento meriterebbe, pertanto, di essere indagato nel lungo periodo, secondo una prospettiva storiografica attenta sia alla dimensione culturale sia alla circolazione del messaggio politico nelle società di massa. La promozione di ricerche sui significati che l' «universo semiotico» della politica attribuisce alle differenze di genere nello spazio pubblico, capaci di attingere all'ampia riserva di suggestioni e metodologie prodotta dagli Studi Culturali e, dunque, di attribuire valore e senso all' iconografia ${ }^{11}$, potrebbe costituire un valido supporto alla costruzione di società più inclusive, ed essere, al contempo, occasione di approfondimento su un sintomo tanto persistente e radicato qual è la disparità di genere nella rappresentanza, e più complessivamente nei ruoli apicali: sfida considerata ineludibile e prioritaria dall' Unione Europea. Gli stereotipi di genere non costituiscono una semplice, lontana astrazione, hanno un impatto sulla vita quotidiana delle persone, riproducono e perpetuano la discriminazione con esiti negativi sull'accesso alle carriere politiche (e non solo), sui successi e sugli insuccessi dei soggetti in carne ed ossa. Sono anche veicolo di messaggi che concorrono all'orientamento dell'elettorato e intervengono sulla stessa attrattività della politica.

Nelle pagine che seguono, senza la minima pretesa di esaustività o di completezza, mi propongo di offrire alcune tessere sull'immagine dei parlamentari nei primi vent'anni anni di storia della Repubblica Italiana. L'arco cronologico prescelto va dal conseguimento del suffragio femminile attivo e passivo (1945-1946) e la IV Legislatura (1963-1968). Questa pe-

8. S. B. Kaiser, "La politica e l'estetica dello stile delle apparenze. Prospettive moderniste, postmoderniste e femministe”, en P. Calefato (Dir.), Moda \& Mondanità, Bari, 1992, 165-194, la cit. è a p. 168.

9. E. Giomi, "Da Drive in alla Makeover Television. Modelli femminili e di rapporto fra i sessi nella Tv berlusconiana (e non)", Studi culturali, 1, 2012, 3-27, la citazione è a p. 23. Si vedano anche G. Sensales, A. Areni, A. Del Secco, "Le ministre del centro-sinistra (2006) e del centro-destra (2008) nella stampa italiana: comunicazione politica e rappresentazioni di genere", Psicologia sociale, 2, 2012, 203-230; D. Brancati, Occhi di maschio. Le donne e la televisione in Italia. Una storia dal 1954 a oggi, Roma, 2011.

10. G. L. Mosse, La cultura dell'Europa occidentale nell'Ottocento e nel Novecento, Milano, 1986.

11. P. Burke, La storia culturale, Bologna, 2009; Id. Testimoni oculari. Il significato storico delle immagini, $2^{\mathrm{a}}$ ed., Roma 2017. 
riodizzazione matura da due principali considerazioni. Il termine a quo, 1945-46, indica l'avvento sul palcoscenico politico italiano dell'elettrice e dell'eletta, e la nascita della Repubblica. A quella data, precisamente nel mese di settembre, 13 donne (su 304 membri iniziali poi 430) per la prima volta sono nominate in un' istituzione nazionale, la Consulta, organo provvisorio e non elettivo. L'anno successivo, in occasione delle elezioni amministrative, 2000 elette entrano nei Consigli comunali; il 2 giugno 1946, data del Referendum istituzionale e dell'elezione della Costituente, 21 donne (su 556 membri) siedono in Assemblea. Alle elettrici e alle elette si volge lo sguardo dei partiti politici decisi a conquistare l'elettorato; dell'associazionismo femminile impegnato a formare «la cittadina»; dei media che informano ed orientano la grande massa degli elettori. Il termine ad quem, 1968, invece, oltre a coincidere con la fine della IV Legislatura, marca la chiusura di una stagione politica. Dalla fine degli anni Cinquanta e lungo il successivo decennio, malgrado le discontinuità e le congiunture più o meno favorevoli, il Paese ha attraversato «la grande trasformazione» che ha massicciamente investito la dimensione strutturale, sociale e culturale. Non senza limiti e contraddizioni, anche gravi, l' Italia da paese agricolo è diventato un paese industriale, i consumi sono cresciuti e con essi nuovi stili di vita e nuove aspirazioni; i giovani hanno acquisito una visibilità inedita, che avrà sbocco nell'eccezionale protagonismo del '68. La modernizzazione interviene pure sul genere: milioni di donne, anche in seguito alla scolarizzazione che investe il mondo femminile, alle trasformazioni innescate dal «boom economico» tra il '50 e il '60 sono sempre più visibili nello scenario pubblico; occupano il settore industriale, il terziario, il mondo delle professioni. Si approvano importanti leggi che intervengono sulla dimensione pubblica e privata. Matura una nuova consapevolezza che, ai margini degli anni Sessanta, insorge nei movimenti femministi, per molti versi, portatori di istanze lontane dalla tradizione dell'associazionismo di matrice laica e cattolica. Non è certo questa la sede per esaminare l'identità e il ventaglio di posizioni espresse da questo nuovo soggetto politico ma, ai fini del discorso affrontato in queste pagine, conviene ricordare che il femminismo abbandona l'orizzonte dei diritti per rivolgersi soprattutto alla dimensione privata, alle relazioni interpersonali; si colloca al di fuori e «autonomamente» dai partiti, mentre la rappresentanza perde centralità e valore. Una scelta che marca una svolta, se non addirittura una cesura, nella storia dei movimenti delle donne che, fin dalle origini, avevano posto al centro della propria agenda il tema dei diritti. Il «nuovo femminismo» rifiuta questa impostazione e si pone oltre la politica istituzionale. Dal 1945 al 1968, quindi, si assiste alla parabola dell'eletta che passa da catalizzatore, seppure sovente in senso negativo, della stampa e dell'opinione pubblica, a soggetto trascurabile. Un dato confermato dalle stesse cifre percentuali che indicano, dopo un lieve aumento nel 1948, una flessione fino al 1972.

La cesura, però, non riguarda solo il genere ma tocca la società italiana nel suo complesso che vede rapidamente cambiare le coordinate politiche di riferimento. Dal dicembre del 1969, con la strage di Piazza Fontana, l'Italia vive una grave crisi politico istituzionale. L'ondata del terrorismo investe istituzioni e società. Sebbene si manifestino inedite forme di partecipazione democratica - nelle scuole, nelle università, nelle fabbriche e sul territorio - finalizzate al rinnovamento del paradigma della cittadinanza, la democrazia e lo stesso Stato sono in pericolo. 
Il presente contributo si incentra su questo primo ventennio di storia della Repubblica Italiana ma, al fine di avviare una prima riflessione su una delle tante eredità che ricadono sulla giovane democrazia, che non può prescindere da una vocazione paritaria e, dunque, dalla definizione di un paradigma della cittadinanza attento alla dimensione di genere, si è scelto di soffermarsi sinteticamente su due categorie che possono essere considerate antenate di quella della «eletta» o tout court della «donna politica». La prima è la «femminista», soggetto che acquista una inedita visibilità tra età giolittiana e primo dopoguerra, fase che vede un articolato dibattito nella società, nei partiti, nel Parlamento sulla cittadinanza femminile; la seconda è la «sovversiva» - negli anni del fascismo il termine indica diverse appartenenze - che si colloca oltre i modelli di genere funzionali alle politiche di nazionalizzazione delle masse. Se la femminista rappresenta una minaccia al sistema di genere e, di conseguenza un pericolo per l'ordine sociale e politico, la «sovversiva» è una nemica dello Stato e, in quanto tale, un pericolo per la stabilità di ruoli e modelli ben codificati dal totalitarismo fascista che, pur con le evidenti contraddizioni sottolineate da Victoria De Grazia ${ }^{12}$, attua specifiche politiche, compresa la repressione, al fine di mantenere salda la divisione di genere. Le «femministe» e le «sovversive» con la loro azione mettono in discussione la linea di demarcazione tra sfere di competenza maschili e femminili definite sulla base del «naturale» principio che vuole le donne mogli e madri, affidando loro la dimensione privata e al genere maschile quella pubblica ${ }^{13}$.

\section{I precedenti}

Nel 1918 chi si apprestava a leggere il nuovo libro di Teresa Labriola, I problemi sociali della donna, poteva imbattersi in un'annotazione critica sul maschilismo in Italia: «Anche ora durante la grande rivoluzione delle nazioni civili, si osserva l'indifferenza degli uomini verso le donne, in quanto forze sociali». L'indifferenza - che l'intellettuale femminista giudicava «sommamente pericolosa» - era sostenuta da un «atteggiamento ironico e critico degli uomini italiani verso le poche donne organizzatrici, conferenziere, scrittici di questioni sociali» ${ }^{14}$. Sarcasmo e denigrazione sono diffuse dalla satira che testimonia ed alimenta un immaginario discriminante con una galleria di femministe lontane dal canone della bellezza e, in questo gineceo, abitava pure Teresa Labriola ${ }^{15}$. Lo stereotipo della femminista, non troppo lontano da quello dell' intellettuale, o meglio, della donna nuova, vero e proprio emblema del «primo

12. V. de Grazia, Le donne nel regime fascista, Padova, 1993. Sul tema si veda anche: L. Branciforte, Donne in onda nel ventennio fascista tra modernità e tradizione (1924-1939), Soveria-Mannelli, 2018.

13. C. Pateman, The disorder of women. Democracy, feminism and political theory, Oxford, 1989; Ead. Il contratto sessuale, Roma, 1997.

14. T. Labriola, I problemi sociali della donna, Bologna,1918, 56-57.

15. Il futuro del Parlamento Illustrato: «Nel giorno della mùliebre vendetta, allor che andran le donne in Parlamento e i maschi faranno la calzetta, avrà su tutte l'altre il sopravvento, e chiederà più spesso la parola, l'avvocata TERESA LABRIOLA». 
femminismo», possiede un corpo sproporzionato e sgraziato, tratti somatici irregolari tanto da contraddire l'identità di genere. Gli uomini non possono provare alcuna attrattiva, da qui l'epiteto di «zitelle». L'espressione è densa di significati negativi che vanno dalla frustrazione per il fallimento esistenziale all' invidia, fino all' isteria. L'attacco alle «forze sociali» femminili doveva essere quotidiano e prevedibile se la socialista Luisa Draghi, ne I dieci comandamenti del socialismo, suggeriva alle sue compagne: «sprezza chi ride vedendoti un giornale fra le mani e chi t'insulta se accorri ad una conferenza» ${ }^{16}$. Le donne che si interessavano alla politica, leggono un giornale o partecipano ad una conferenza erano biasimate, colpevoli della decadenza e responsabili dell'alterazione dell'ordine sociale, a cominciare dalla rovina della propria famiglia. Da qui una serie di caricature di donne alla scrivania e di bambini sofferenti $\mathrm{o}$ in pericolo di vita; case abbandonate all incuria, madri che partecipano a comizi e incontri; interni domestici irriconoscibili a causa del disordine che vi regna, spesso trasformati in luoghi di dibattito politico ${ }^{17}$. Per converso, si pubblicano schizzi e disegni di uomini costretti a svolgere i lavori domestici ${ }^{18}$; di mariti maltrattati, dileggiati, non considerati, la cui condizione è proiettata su corpi de-virilizzati ${ }^{19}$.

Nella storia dei primi movimenti femministi, il Primo Congresso nazionale della donna italiana del 1908 - che rappresenta al contempo il momento di massima espansione e di crisi del movimento - viene consapevolmente trasformato dalle promotrici in una sorta di banco di prova, si direbbe, di autorappresentazione collettiva. Un aspetto sul quale, alla metà degli anni Ottanta, era intervenuta, con largo anticipo rispetto agli sviluppi della storia politica in Italia, Paola Di Cori ${ }^{20}$. La spettacolarizzazione dell'evento ingaggiata dalle femministe, possibile grazie alla presenza di personaggi noti e alla solennità dei luoghi dove si svolse l'incontro ${ }^{21}$, sfociano in una campagna di stampa di una certa dimensione accompagnata da una pungente satira che ripropone il canone della inadeguatezza femminile alla «cosa pubblica»: «E se non vi

16. A. Buttafuoco, "Sprezza chi ride". Politica e cultura nei periodici del movimento di emancipazione in Italia, Nuova DWF, 21, 1982, 7-34.

17. GEC (Enrico Gianeri), La donna la moda l'amore in tre secoli di caricatura, Milano, 1942; "Il voto alle donne", Guerin meschino, 50, 1931; "Se le donne avessero il voto", Guerin meschino, 52, 1923; Guerin Meschino, 45, 1926: «Una madre si rivolge alla figlia, una donna sciatta e sgraziata, seduta alla scrivania "Peccato che una ragazza come te si sciupi con la politica invece di prendere marito"».

18. "La tribuna delle signore alla Camera, mentre si discute sul voto alle donne", Corriere della Sera, 29 marzo 1925.

19.P Morris, F. Ricatti, M. Seymour (Dir.), Politica ed emozioni nella storia d'Italia: dal 1848 ad oggi, Roma, 2012.

20. P. Di Cori, "Il doppio sguardo. Visibilità dei generi sessuali nella rappresentazione fotografica (19081918)", en D. Leoni, C. Zadra (Dir.), La grande guerra esperienze memorie immagini, Bologna, 1986, 765 799, con particolare riferimento alle pp. 776-778.

21. P. Gabrielli, "Congresso del 1908 e dintorni: qualche riflessione sul "fare politica" delle donne", Storia e problemi contemporanei, 49, 2008, 5-24. 
si concedesse niente, come vi vendichereste? - Ingrandendo i nostri cappelli da teatro!!» ${ }^{22}$. Luigi Lucatelli faceva recitare a un suo personaggio: "Casa mia non è più una casa, ma bensì un antipasto di rivoluzzione sociale, come ché si è costituito un commitato fra Terresina, la moglie del pizzicagnolo in faccia e la signora Gertrude di sopra. Terresina non abbada più alle faccende! L'altro giorno il riso aveva bollito tanto che era diventato una colla e quando ce lo dissi sa che mi arispose?... Dice: tanto meglio, ci attaccheremo i manifesti del partito! ${ }^{23}$.

L'esposizione delle donne nel corso della Grande guerra, il senso di responsabilità, i sacrifici, le capacità dimostrate nel sostegno al fronte interno, tutte questioni abbondantemente presenti nei tanti appelli suffragisti del dopoguerra, non valsero a modificare le rappresentazioni (e non solo). Del resto la «cultura di guerra» aveva per molti versi ristabilito con successo il predominio del materno, fosse pure sotto la veste della crocerossina o della mater dolorosa. L'Angelo bianco, al lavoro tra feriti e mutilati, che guida e protegge i soldati disabili, diviene una vera e propria icona, incarnazione della purezza e della virtù. Accanto a lei ci sono la vedova o la madre in gramaglie pronte ad immolare marito e figli alla patria in guerra. Speculare a queste figure è colei che spende in beni di consumo, che pratica il demonizzato lusso, mentre i suoi connazionali muoiono al fronte. Nel dopoguerra, in un' Italia colpita dai lutti, dai pesanti sacrifici, dilaniata da una grave crisi politica e sociale, si riaccende il dibattito sul voto, e, nonostante l' impegno speso per «la patria in guerra», sulle femministe in primis, continuano a fioccare accuse. Congegni linguistici e visivi le abbinano alla leggerezza, alla futilità, alla inadeguatezza ed il suffragio diventa, ancora una volta, l'emblema del pericolo. Molteplici gli interventi sulla proverbiale frivolezza e sulla lussuria incarnate in calze di seta, pellicce, estrosi cappelli; oggetti inutili, superficiali per i quali si dilapidano fortune ${ }^{24}$. L'estetismo, sintomo di degenerazione, si fa traslato dell'insensatezza femminile, così come la vanità. Merita citare almeno la vignetta che vede donne pronte a rinunciare al suffragio pur di non dichiarare di avere raggiunto la maggiore l'età ${ }^{25}$. Ricorrenti sono pure incapacità, volubilità o instabilità che, sommati e amalgamati insieme alla lussuria, rimandano alla debole o inesistente eticità. Facendo leva sulla retorica del rovesciamento, una rivista satirica affermava: «La politica è l'arte della menzogna. Ora, non esiste al mondo essere vivente che sappia mentire più della donna». Oltre ad essere scaltre e bugiarde, esse sono pure amanti dell'ozio considerato causa di corruttibilità: «La politica è l'arte del dolce far niente. Ebbene, ma mi sa trovare una specie umana o zoologica che più della donna possegga in

22. Cirillo, "Dopo il Congresso", Pasquino. Rivista umoristica della settimana, 1908. Tra le altre pubblicazioni vi sono le caricature delle convegniste.

23. Ficcanaso, "Spigolature di ilarità. Ai tempi delle suffragette", La Tribuna illustrata, 4 marzo 1945. Il testo volutamente presenta grossolani errori ortografici a dimostrare la diffusione del femminismo tra le classi meno abbienti e poco alfabetizzate.

24. "Numero" 1919, In tema di elezioni. Una giovane chiede all'altra: "Come giustificherai questa fantastica somma spesa in calze?" La risposta: "Propaganda elettorale".

25. "Il voto alle donne", Pasquino. Rivista umoristica della settimana, 24 giugno 1923. 
sommo grado questa qualità? $»^{26}$. La presenza di corpi femminili nella sfera politica abitata al maschile e idealizzata come neutra e desessualizzata rimanda alla promiscuità, dunque, alla sessualizzazione di quello spazio, che trova espressione nella erotizzazione della donna politica. Questo processo ha un medium visivo nella corruzione prodotta dallo stesso genere femminile dotato di una forte carica erotica e sessuale. L'avvenenza fisica, intorno alla quale si tesse una antica narrazione che le conferisce il potere, specifico e malefico, di soggiogare gli uomini ${ }^{27}$, dà luogo alla lascivia e all' inganno, cause del ridimensionamento del potere maschile, minaccia per la morale sessuale e per l'etica.

Le politiche di nazionalizzazione delle masse veicolano modelli omologati, esaltano l'uomo e la donna dello Stato fascista, coloro che si posizionano oltre questa orbita sono considerati «nemici», resi «invisibili» dalla cancellazione della libertà di espressione oltre che dalla condanna al carcere o al confino. Sono visibili, invece, nella documentazione prodotta dalle istituzioni addette al controllo degli oppositori, una fonte preziosa pure per lo studio delle rappresentazioni. Socialiste, comuniste, anarchiche, femministe, per gli «occhi del potere» sono soggetti irrequieti e malvagi, arroganti e prepotenti, trascurano il focolare con gravi conseguenze sugli assetti familiari e sociali. Le «sovversive» sono sfrenate, sono pericole, sono simili alla "popolana ribelle» di Michelle Perrot ${ }^{28}$ e alla «rossa sguaiata» di Joan $\operatorname{Scott}^{29}$, che nell'Ottocento occupano con disinvoltura lo spazio urbano, le vie e le piazze così come i luoghi di raduno considerati maschili e pericolosi, quali erano le sedi del movimento operaio. La loro mobilità, imprescindibile dalla propaganda, che si svolge in luoghi diversi, e dalla clandestinità, che impone continui cambiamenti di residenza, testimoniano una libertà di movimento interdetta alle donne.

La tematica spazio-tempo, al centro di riflessioni teoriche e studi, si presenta in questo caso quale fertile terreno per comprendere - per dirla con Erving Goffman - l' «orchestrazione» dei rapporti di genere ${ }^{30}$. L'andatura veloce, il portamento sicuro, l'eleganza o la grossolanità nelle movenze, funzionali all'identificazione, hanno anche lo scopo di rafforzare lo stereotipo negativo.

La scelta della militanza politica, interpretata come una drastica rottura della tradizione, genera un modello che include nelle sue faccettature, presunzione, fanatismo, inganno, immoralità. Eclatante il caso di un funzionario che, lasciando affiorare un sentimento di disprezzo, accusava una detenuta politica di una colpa imperdonabile per la morale

26. "La donna si dà alla vita pubblica", Pasquino. Rivista umoristica della settimana, 1 aprile 1923.

27. Ricco di sollecitazioni in tal senso B. Vinken, "Moda e identità", en B. Valli, B. Barzini, P. Calefato (Dir.), Discipline della moda. L'etica dell'apparenza, Napoli, 2003, 193-204.

28.M. Perrot, "La femme populaire rebelle", en Ch. Dufrancatel (Dir.), L'Histoire sans qualités, Paris, 1979, 123-156.

29. J. W. Scott, “Louvriére mot impie, sordide”... Le discours de l'Economie politique francaise sur les ouvrieres 1840-1860", Masculin-Feminin-1, 83, 1990, 2-15. Sulla stigmatizzazione delle "rivoluzionarie" P. Gabrielli, Fenicotteri in volo. Donne comuniste nel regime fascista, Roma, 1999; E. Bini, "Le pétroleuses: corpi di donne in rivolta", Genesis, 2, 2006, 193-216.

30. E. Goffman, “The arrangement between the sexes”, Theory and Society, 4, 1977, 301-331. 
dell'epoca, quella di «trascura[re] i doveri della famiglia» e, per questo motivo, la bollava con il marchio infamante di «donna di poco riguardo» ${ }^{31}$. La mancata assunzione di questo dovere-responsabilità, intorno al quale si codificano i ruoli e le sfere di competenza tra i sessi, vale a dire ciò che è lecito e illecito per le donne e per gli uomini, e si struttura l'identità di genere, trasforma coloro che oltrepassano i confini assegnati al proprio genere, scegliendo la politica rispetto alla famiglia, il pubblico al privato, in un soggetto pericoloso ed ambiguo, dotato di un carisma per certi versi distruttivo:

Atteggiandosi a protettrice delle compagne è riuscita ad accattivarsene l'animo in modo che ad un di lei cenno sarebbero capaci di commettere qualunque disordine. Recentemente è bastato che rivelasse rancore verso una compagna minacciandola di schiaffi, perché costei fosse colpita a sangue da sei energumene, mentre si trovavano a passeggio ${ }^{32}$.

La «sovversiva» esprime una forza contro natura, passionale, violenta, distruttiva, travolge l'ordine stabilito sia nella sfera pubblica - dove si erge a capo e fomenta la rivolta - sia in quella privata - dove viene meno ad uno dei principali doveri assegnati alle donne, quello di madre. In altri casi tale carica negativa trova una proiezione nel potere seduttivo, subdolo e pericoloso: «Nata e cresciuta in ambiente torbido, ha ereditato dal proprio genitore degenerato e sovversivo, i peggiori sentimenti. Esercitava la prostituzione dandosi di preferenza a militari onde aver modo di professare fra di essi le proprie idee» ${ }^{33}$. Da queste tare traeva alimento lo spettro del rovesciamento del potere politico e di quello patriarcale: «Di carattere piuttosto violento, domina il temperamento del marito e del suocero. Si è vantata più volte pubblicamente di saper maneggiare bene la rivoltella e di non temere nessuno [...]. Non si può escludere che sia capace di commettere delitti, specialmente politici» ${ }^{34}$. Il richiamo all'uso delle armi, proiezione e prerogativa della maschilità, si afferma alla stregua di un congegno retorico capace di suscitare dubbi circa l'autenticità dell'appartenenza di genere e degli orientamenti sessuali del soggetto ${ }^{35}$.

A questa tipologia femminile di donna poco onorevole, corrisponde la definizione di un preciso modello maschile difforme dal canone della mascolinità egemone ${ }^{36}$. Vagabondo, violento, impulsivo, ma non irrazionale, è infatti il «sovversivo». La sua pericolosità è data

31. Archivio Centrale dello Stato (Roma) (d'ora in avanti ACS), Ministero dell'Interno, Direzione Generale di Pubblica Sicurezza, Casellario Politico Centrale, (d'ora in avanti CPC), b. 2370, Lea Giaccaglia, Regia Prefettura di Bologna, 9 novembre 1931.

32. ACS, CPC, b. 2370, Lea Giaccaglia, Regia Prefettura di Bologna, 9 novembre 1931.

33. ACS, CPC, b. 514, Egle Gualdi, Telegramma espresso di Stato, Divisione Carabinieri Regi, Reggio Emilia, al Comando Arma dei Carabinieri, 24 dicembre 1926.

34. ACS, CPC, b. 2206, Vanda Fuschini, Regia Prefettura di Forlì, Forlì 4 agosto 1930.

35. J. B. Elshtain, Donne e guerra, Bologna, 1991. Per una diversa rappresentazione della ribelle si veda C. Papa, “Torino 1917: le donne fra tumulto e rivoluzione”, en G. Allegri, A. Longo (Dir.), Rivoluzione fra mito e costituzione. Diritto, società e istituzioni nella modernità europea, Roma, 2017, 243-262.

36. R. W. Connell, Maschilità. Identità e trasformazioni del maschio occidentale, Milano, 1996. 
dalla capacità di agire, quindi, di scegliere. Tutti cliché che ritroviamo in abbondanza nella letteratura, sorgente alla quale attingere ritratti di operai pigri, alcolizzati, farabutti, sensuali, criminali, figure quanto mai lontane dalla laboriosità e dall'equilibrio elevati a codici di riconoscimento e di autorappresentazione della borghesia ${ }^{37}$. Il decentramento rispetto all' immagine codificata rende questi uomini insolvibili rispetto ad uno dei principali dover loro assegnati, qual è garantire l'onore delle donne. Essi, per molti versi, possono definirsi speculari all' immagine della sovversiva.

\section{3. «Deputatesse» e deputati della Repubblica}

Nel 1945 il suffragio, insieme con la destabilizzazione dei ruoli di genere causata dalla guerra totale, genera il fantasma del «disordine» veicolato dalla efficace metafora del «salto nel buio». In agguato c'era allora, come già nel passato, il timore del rovesciamento dei ruoli e del potere. Come egregiamente sentenzia ancora nel 1948 la «La Tribuna illustrata»: Donne alla camera: vuoto in cucina ${ }^{38}$.

A ridosso del conflitto bellico si definiscono le forme del nuovo stato italiano e del governo, si sceglie la nuova classe politica, si plasma il volto della nuova repubblica italiana. Lungo questo crinale si colloca la definizione del nuovo ceto politico e il suo accreditamento elettorale che, nelle società di massa, transita non esclusivamente in strategie e programmi ma in simboli, miti, leader, sui quali si fondano e dai quali traggono linfa le religioni politiche ${ }^{39}$. Sempre in questa fase si riattiva il nesso tra corpo della nazione e corpo delle donne, basti pensare all' Italia turrita o alla bella fotografia di Federico Patellani che affida ad una giovane donna dai tratti considerati tipici della bellezza mediterranea, l'incarnazione della Repubblica Italiana. Esemplare, tra le altre, una vignetta che riferendosi alla prima notte di nozze, ritrae in una camera da letto con tappezzeria tricolore, una giovane Italia che, seduta su un letto, si scioglie i capelli e poco lontano da lei, il Presidente della Repubblica si sveste ${ }^{40}$. Le grandi trasformazioni successive alla Liberazione investono pure la definizione dell' uomo politico, un soggetto all'altezza dei tempi capace di incutere fidu-

37. O. Blanc, "Femmes et Révolution, l'exemple d'Olympe de Gouges", Cahier du GRHIS, 17, 2006, 53-63.

38. La duchessa minima, "Donne alla Camera vuoto in cucina", La Tribuna illustrata, 16 maggio 1948.

39. Sul tema delle religioni politiche, sia in Italia sia all'estero, si dispone di un'ampia bibliografia, hanno rappresentato un apripista in questo ambito gli studi di Emilio Gentile, si veda tra gli altri Il culto del littorio. La sacralizzazione della politica nell'Italia fascista, Roma-Bari, 1993 e Le religioni della politica fra democrazie e totalitarismi, Roma-Bari, 2001. Per l'Italia repubblicana M. Ridolfi, Propaganda e comunicazione politica: storia e trasformazioni nelletà contemporanea, Milano, 2004 e Italia a colori. Storia delle passioni politiche dalla caduta del fascismo ad oggi, Firenze, 2015. Sull'avvento della società di massa e nuove forme della politica influenzate dalla cultura commerciale, resta fondamentale M. Y. Ostrogorski, Democrazia e partiti politici, a cura di G. Quagliarello, Milano, 1991.

40. "Prima notte", Il Travaso, 7 luglio 1946. 
cia e rispetto, di rispecchiare soprattutto i modesti cambiamenti in atto nel Paese, lontano dalla invadente, tracotante virilità del maschio fascista incarnata in primis da Mussolini ${ }^{41}$.

È nell' immediato dopoguerra, infatti, che riecheggia con maggiore insistenza l'antitesi normalità-anormalità: una polarità di lungo periodo - come si è accennato -, un'utile unità di misura per classificare soggetti e definire modelli, compiti, funzioni, comportamenti con le loro coerenti aspettative sociali. Dal profondo pozzo di stilemi e immagini, un'ampia e ricca riserva di "costruzioni» funzionali a vecchie gerarchie e asimmetrie, si attinge per definire l'antitesi normalità-devianza, per ribadire la «naturale estraneità» delle donne alla dimensione pubblica perché prive di equilibrio, saggezza, attitudine alla scelta e, dunque, propensione alla responsabilità individuale e collettiva. La contaminazione e la fluidità dei ruoli sono i temi massicciamente utilizzati per lanciare l'allarme su «un mondo alla rovescia», per richiamare l'attenzione sulla virilità minacciata dalla invasione di un agente estraneo. La caricatura politica - un genere sostanzialmente diretto ad un pubblico maschile e alla dimensione emotiva - pur nell'esagerazione che le è propria, si rivela una fonte fruttuosa per cogliere gli umori più profondi del Paese. Le pagine satiriche ricompongono e restituiscono, seppure nella deformazione e nell'amplificazione, i tratti della mentalità dell'epoca, i preconcetti e i luoghi comuni più diffusi che, in altra veste, ritroviamo pure in altri generi. É il caso di alcuni rotocalchi, che pubblicano fotografie e commenti stereotipati. "Oggi» sosteneva che democristiani e comunisti avevano conquistato il consenso femminile in cambio di calze di nylon e di seta ${ }^{42}$.

In qualche caso, si avvicina al canone stigmatizzante la documentazione prodotta dalle istituzioni, come conferma il dibattito alla Costituente sulle professioni femminili, compresa la magistratura, nel corso del quale gli oppositori ricorrono ad un concentrato di luoghi comuni, ai binomi antitetici ragione-sentimento, resistenza fisica-debolezza» come nel caso del democratico cristiano Giuseppe Cappi:

Il dovere di dire che una delle ragioni principali per cui ha espresso parere contrario all'ammissione delle donne nella magistratura è che, almeno oggi, nella coscienza popolare non vi è la convinzione che le donne possano esercitare - soprattutto indiscriminatamente - la funzione di giudice. Si dovrebbe fare, semmai, un referendum; in ogni modo riassume la ragione della sua opposizione in questa proposizione: a suo parere, nella donna prevale il sentimento al raziocinio, mentre nella funzione del giudice deve prevalere il raziocinio al sentimento. ${ }^{43}$

L'esempio più eclatante del persistere di una cultura arretrata basata sulla disparità di genere è quello di Enrico Molè, della Democrazia del lavoro, che sosteneva: «E” soprattutto

41. L. Passerini, Mussolini immaginario, Roma-Bari, 1991.

42. "Calze alle elettrici", Oggi, 21 maggio 1946.

43. Atti dell'Assemblea Costituente, Commissione per la Costituzione, Assemblea Plenaria, resoconto della seduta pomeridiana di venerdì 31 gennaio 1947, 26, G. Cappi, 263. 
per i motivi addotti dalla scuola di Charcot, riguardante il complesso anatomo-fisiologico, che la donna non può giudicare» ${ }^{44}$.

La sessualizzazione dello scenario pubblico, la promiscuità dei generi, data la sua attitudine a trasporre la relazione tra corpo e politica, corruzione sessuale e corruzione politica, è largamente presente, incarnata da donne discinte nell'abbigliamento, con le zone erogene sottolineate. Soggetti che evocano lo spettro della lascivia, della lussuria femminile con i suoi catastrofici effetti sulla morale pubblica. "Giovanna la compagna» possiede un corpo eroticizzato, indossa un abito attillato e corto, fuma e dichiara con strafottenza a un probabile marito che non sarà più «tutta sua» ma del "popolo» ${ }^{45}$. Non lontana da questa caricatura una giovane donna, provocante e in abiti discinti afferma: «mio marito mi proibisce di presentarmi come candidata al parlamento. E pensare che invece ho tanta disposizione a fare la donna pubblica ${ }^{46}$. L'attrazione erotica si conferma quale temibile tentazione per gli uomini, causa di infantilizzazione e regressione da cui scaturiscono oziosità, imbecillità, sciocchezza, inidoneità. Insomma si trasferiscono sul maschile le cosiddette prerogative femminili.

La definizione di un'estetica femminile (e maschile), dunque, interseca il «discorso» politico, pertanto, individuare il grado di condivisione, rifiuto, manipolazione dei canoni estetici può aiutare nella decodificazione degli stereotipi di genere in politica.

La figurina smaliziata, spesso provocante, dalle zone erogene delineate, in pose scomposte, di bella presenza convive con il suo contrario. Se in alcuni casi si sottolinea l'avvenenza delle deputate, tanto da citare le loro chiome bionde, nominarle Miss Parlamento, soprattutto ad essere deplorata è l'assenza di qualità estetiche che sconfina nella sgradevolezza o, addirittura, nell'animalità, secondo una costruzione radicata e storicamente sperimentata nel discorso politico finalizzata alla definizione della antitesi amici-nemici, superiori-inferiori, estraneità-attinenza ${ }^{47}$. La bruttezza, frutto della morbosità, rimanda all' indissoluto, all'illecito, a «caratteri degenerativi», tra i principali l'invidia, "passione deformante e velenosa che viene scatenata nelle donne dalla bellezza dell'altra, e finisce per ritorcersi contro loro stesse deturpandole nel corpo e nell'anima» ${ }^{48}$. Questo stereotipo ha una sua appendice nell'inversione di genere. La donna politica assume la veste di un soggetto dai tratti virili. Lineamenti pronunciati, accenni di barbe, toraci villosi, peluria diffusa, corpo robusto spesso muscoloso, uso della sigaretta, pose sgraziate, le conferiscono un'identità sessuale incerta. La questione delle virtù fisiche femminili storicamente si in-

44. Atti dell'Assemblea Costituente, Commissione per la Costituzione, Assemblea Plenaria, resoconto della seduta pomeridiana di venerdì 31 gennaio 1947, 26, E. Molè, 263.

45. http://www.eletteedeletti.it.

46. Vignetta, Cantachiaro, 14 dicembre 1945.

47. A. Ventrone, Il nemico interno. Immagini e simboli della lotta politica nell' Italia del '900, Roma, 2005; F. Cammarano, S. Cavazza (Dir.), Il nemico in politica. La delegittimazione dell'avversario nell'Europa contemporanea, Bologna, 2010.

48. E. Pulcini, "Specchio, specchio delle mie brame..." Bellezza e invidia, Firenze, 2017, 23; E. Pulcini, Invidia. La passione triste, Bologna, 2011. 
terseca - come suggerisce Stephen Gundle - con la costruzione dell' identità nazionale ${ }^{49}$, le deputate contraddicono la rappresentazione dominate sulla «bella italiana». Esse rientrano - come le antenate impegnate in politica - in un modello indesiderabile e si riaffacciano le «zitelle», ed è solo in virtù di tale deplorevole condizione che le donne si occupano di politica, nell'attesa e nella speranza di trovare un buon marito.

Il messaggio sulla presunta insufficienza di doti pubbliche è rafforzato dall' insistente richiamo all'estetismo: la bramosia di possedere abiti, cappelli, pellicce, gioielli, è uno dei leit motiv - si è già detto - della satira politica, ma nel secondo dopoguerra, il messaggio, espresso con formule differenti, trova spazio in diverse sedi, quotidiani e riviste. Fino dalla loro prima apparizione ufficiale, sulle Costituenti fioccano considerazioni sul corredo vestimentario. Si commentano tagli di abiti, colori, cappelli, ventagli, acconciature, tutti oggetti che intervengono sullo «stato grezzo» di una donna per restituire una femminilità manipolata ${ }^{50}$. La relazione tra politica, estetica, genere - come diversi studi hanno messo in luce - trasferisce sul corpo femminile le appartenenze sociali e politiche ${ }^{51}$.

Tra il 1945 e il 1948 frivolezza e civetteria si danno la mano, esaltate da una serie di commenti sul look, secondo una pratica discorsiva finalizzata a ribattere la costruzione che vuole le donne dedite in maniera superficiale alla moda e riconduce, proprio evocando il permanere di antichi stereotipi, la novità del protagonismo femminile nell'alveo della tradizione. Si depotenziava in tal modo - come denunciava la giornalista Anna Garofalo e con lei una rivista femminile - professionalità ed esperienza politica ${ }^{52}$. L'attrattività del messaggio era amplificata e rafforzata dalle note sui colori. Nero, beige, rosso, giallo, marrone, rosa, turchino, viola; pois e fiori, merletti e stoffe vaporose, fiocchi e colletti erano i componenti di una disomogenea, persino disordinata, immagine, cui si contrapponeva quella dell'austero parlamentare, con un corpo raccolto in un elegante completo scuro sempre accompagnato dall' impeccabile cravatta, secondo uno stile fedele all' imperativo: «niente fuori posto». Nei primi anni della Repubblica, gli eletti non sono oggetto di insistenti osservazioni. Tutt'al più, si può dare risalto a qualche particolare, quale il basco di Nenni, cappello largamente diffu-

49. Il riferimento è a S. Gundle, Figure del desiderio. Storia della bellezza femminile italiana, Roma, 2007. Sullo stesso discorso vedi C. Forth, Masculinity in the Modern West: Gender, Civilization and the Body, Basingstoke, 2008; C. Fletcher, S. Brady, R. Moss and L. Riall (Dir.), The Palgrave Handbook of Masculinity and Political Culture in Europe, Basingstoke, 2018.

50. C. Evans, M. Thornton, Women and Fashion: a new look, London, 1989, 13: «La storia della donna assume una forza speciale quando la si osserva attraverso lo sguardo sul corpo nudo e vestito. La trasformazione, significante forte per la figura femminile, s'inscrive in mutazioni stilistiche che parlano di un cambiamento in ambito sociale e culturale».

51. B. Vinken, "Moda e identità", en B. Valli, B. Barzini, P. Calefato (Dir.), Discipline della moda. L'etica dell'apparenza, Napoli, 2003, 193-204, la citazione è a p. 194. Su questi processi storici fondamentale G. L. Mosse, Sessualità e nazionalismo. Mentalità borghese e rispettabilità, Roma-Bari, 1984. Si veda anche E. Mora, "La moda italiana dell' uomo integrato", en E. dell'Agnese, E. Ruspini (Dir.), Mascolinità all'italiana. Costruzioni, narrazioni, mutamenti, Torino, 2007, 106-108.

52. A. Garofalo, L'italiana in Italia, Bari, 1956, 45. P. V. S., "La repubblica delle donne ovvero il referendum sul referendum”, Per voi signora. Rivista mensile di moda tessuti e lavori, 1946. 
so nell'emigrazione antifascista e la sua cravatta rossa: oggetti simbolo di un'appartenenza politica. In qualche caso, si cita la trascuratezza. Il comunista Emilio Sereni indossava una camicia color crema aperta; l'azionista Leo Valiani, e qualche altro con lui, si presentavano ai lavori della Costituente con la camicia, certo rigorosamente abbottonata, ma senza cravatta ${ }^{53}$.

La mancanza di decoro più che l'essere fuori moda di alcuni, quasi sempre esponenti della sinistra, trasmette sostanzialmente il loro essere fuori luogo, inadeguati ai compiti che l'incarico pubblico richiede ${ }^{54}$ e denota una debole lealtà verso le istituzioni.

Se questa è la rappresentazione che emerge dalla stampa, estremizzata dalla satira, merita sottolineare che le scelte delle singole deputate presentano un panorama assai meno omologato e uniforme da cui traspare quanto abiti e accessori vengano usati con cognizione nella costruzione della donna pubblica:

La moda portata - osserva A. Carlo Quintavalle - è qualcosa che esula completamente dalla progettazione dello stilista ed anche dalla realizzazione del suo progetto come viene presentata alle sfilate o nelle riviste di moda. La moda portata è un test vero e proprio sulla cultura vestimentaria dei singoli, ma soprattutto è il momento delle proiezioni dei miti dei singoli, dei singoli procedimenti, a livello di scelte dei singoli, in relazione all' immaginario collettivo ${ }^{55}$.

La questione di un consono corredo vestimentario è tutt'altro che ignorata dalle dirigenti consapevoli che «lo stile delle apparenze», inteso quale aspetto visibile delle identità «foggiate tramite l'uso dell'abbigliamento e degli accessori, l'acconciatura dei capelli ed il tipo di trucco e tutti i procedimenti usati per modificare la forma e l'aspetto del corpo ${ }^{56}$, si attesta quale territorio tutt'altro che secondario per la formazione di un'élite politica ${ }^{57}$, tanto da essere considerato da qualcuna fondamentale nella formazione di quadri dirigenti.

I documenti raccolti restituiscono parlamentari che non vestono certo con casualità, come conferma una fotografia che ritrae quelle del Pci uniformate in semplici abiti scuri, a distinguerle solo qualche piccolo discreto fiocco o colletto bianco. In questo caso, facendo propria la tesi di Roland Barthes, circa la distinzione tra costume, corrispondente alla istituzione indipendente dall' individuo, e vestito che rimanda alla realtà individuale, si direbbe che le parlamentari comuniste propendono per il primo ${ }^{58}$. Nell' immediato dopoguerra, la preoccupazione di non «distaccarsi» troppo dallo stile spartano delle donne dei ceti popolari,

53. "Primo giorno della Consulta", Domenica, 30 settembre 1945.

54. A. Lurie, The Language of Clothes, New York, 1981, 34-35.

55. A. C. Quintavalle, "Archivio della moda", en A. Nodolini (Dir.), Brunetta. Moda, critica, storia, Parma, 1981, III-XLII, la citazione è a p. XI.

56. S. B. Kaiser, "La politica e l'estetica dello stile delle apparenze. Prospettive moderniste, postmoderniste e femministe", en P. Calefato (Dir.), Moda « mondanità, Bari, 1992, 165-194, la citazione è a p. 166. Per un quadro sul dibattito sempre della stessa, "Studi culturali e fashion theory", Annali d'italianistica, 24, 2006, 199-209; C. Sorba, "The clothing of politics in XIX-XXth centuries", Contemporanea, 4, 2018, 527-540.

57. A. C. Quintavalle, “Archivio...," op. cit., XI.

58. R. Barthes, Sistema della moda, Torino, 1970. 
come già nel passato, è visibile. Si potrebbe osservare, sulla scorta delle osservazioni di George Simmel, che l'imitazione inverte il senso, si muove verso il basso invece che verso l'alto. Ma tali scelte confermano l'avvio di una ricerca estetica capace di restituire la nuova identità della donna italiana divenuta cittadina e rappresentante della Repubblica. Negli anni successivi il quadro cambia. Esse, al di là delle appartenenze politiche e generazionali, individuano nel completo gonna e giacca l'abito libero dagli «impedimenti» ${ }^{59}$. Affermatosi tra Otto e Novecento come abito "da uso», il tailleur "di stoffa ruvida e resistente, quasi maschile, e dalle tinte per lo più neutre ${ }^{60}$, comincia a diffondersi occupando il guardaroba delle signore della piccola e della media borghesia. Soprattutto il capo diviene un simbolo dell'emancipazione femminile. La sua praticità e semplicità lo rendono apprezzabile agli occhi delle suffragiste come confermano le rigorose giacche e le altrettanto lineari gonne delle divise adottate dalle suffragiste inglesi di primo Novecento.

Se nel 1917 è soprattutto la reinterpretazione di Coco Chanel a renderlo famoso, occorre anche tenere presente che la grande guerra, affidando alle donne nuovi compiti, aveva fatto del completo gonna e giacca la sua fortuna, così come lo farà la seconda guerra mondiale che, date le ristrettezze, consiglia confezioni dalle linee semplici, giacche strette, gonne al ginocchio sui quali intervenire con gli accessori a seconda dell'occasione e l'ora del giorno ${ }^{61}$. A partire dagli anni Cinquanta, il capo si afferma, con le variazioni dettate dalla moda, prima tra tutte il taglio morbido, come «abito della donna politica». Un dato che può essere letto quale manifestazione di un diverso rapporto con lo spazio e dettato dalla volontà di rappresentare il corpo libero da ornamenti che possono anche essere impedimenti, ostacoli al movimento, in sintesi, un modo di a tutelare la propria indipendenza. Il messaggio passa attraverso il corpo, un corpo plasmato da numerosi dispositivi disciplinari ma anche «veicolo del nostro essere nel mondo che lo stare al mondo ci costringe ad aggiustare continuamente ${ }^{62}$. Nel secondo dopoguerra, con maggiore evidenza dalla fine del decennio, il tailleur, appare come l'unico omologabile, o almeno analogo, al classico completo maschile. Nel 1961, la prima Tribuna Politica che vede la partecipazione femminile, le deputate dei diversi schieramenti, appaiono curate nel vestire, ben pettinate e, tra le più giovani, è il caso di Tullia Carrettoni c’è chi sfoggia una mise distinta ed originale. Da queste immagini televisive, da quelle pubblicate sulla stampa, si coglie come molte siano estranee all'omologazione al modello maschile, quanto piuttosto alla ricerca di uno stile proprio ${ }^{63}$.

59. Si vedano le osservazioni di M. A. Trasforini, "Le flâneuses". Corpi spazi di genere fra modernità e post-modernità", Studi culturali, VII, 2, 2010, 239-260, la citazione è a p. 242.

60. A. Gigli Marchetti, Dalla crinolina alla minigonna: la donna, l'abito e la società dal 18 al 20 Secolo, Bologna, 1995, la citazione è a p. 176.

61. S. Gnoli, “Un secolo..., op. cit., 94.

62. C. Guillaumin, "Il corpo costruito", Studi culturali, 2, 2006, 307-342, la citazione è alle pp. 334-335.

63. Di indubbio interesse la vicenda di Adele Bei alla ricerca di un proprio stile, si veda a proposito M. A. Serci, "La sindacalista in abito bianco. Alcune note per una biografia di Adele Bei", en P. Giovannini, B. Montesi, M. Papini (Dir.), Le Marche dalla ricostruzione alla transizione, 1944-1960. Atti del Convegno di studi, Ancona, 1999, 298-331. 
Nel dopoguerra, i deputati, lontani dalle rigide pose dei notabili dello Stato liberale e ancor più decisi a distaccarsi dal machismo fascista, si affidano ad un corredo vestimentario sobrio e rigoroso, contraddistinto però da una certa rigidità. Domina il blu ma presto la «mascolinità vestimentaria» dell'uomo politico affianca questa tinta al grigio nelle sue diverse nuances $^{64}$. Colore per eccellenza della moderazione e della dedizione, l' importanza simbolica dell'abito grigio la conferma Joan Sloan con il suo romanzo, del 1955, «L'uomo dal vestito grigio», la storia di un uomo d'affari americano - poi interpretato nell'omonimo film da Gregory Peck - che affronta con conformità e successo la carriera sacrificando la propria individualità ${ }^{65}$. Se «la moda italiana costituisce uno dei motori dell' innovazione dell' immagine maschile nell' ultima parte del XX secolo», è altrettanto possibile affermare che i consumi in questo settore restano sostanzialmente "convenzionali» ${ }^{66}$. Una tendenza che riguarda a maggior ragione i politici attenti a scrivere con i propri corpi la formalità. La «politica delle apparenze», dunque, toccava (e tocca) da vicino gli uomini. Come sottolinea Roger Chartier, la corporeità maschile è un dispositivo capace di «fabbricare il rispetto e la sottomissione, per produrre una costrizione interiorizzata che si rende necessaria in ogni società civilizzata, dove non sia possibile esercitare una violenza immediata degli uni sugli altri ${ }^{67}$. Fasciati in una sorta di «uniforme» che «rende manifesta la rinuncia dell'uomo a un'apparenza vistosa, non degna di lui e nasconde il corpo individuale sotto un anonimo abito «funzionale» $\mathrm{i}$ parlamentari addottano un look atto ad affermare il «potere maschile disincarnato, che di fatto coincide simbolicamente con il potere esercitato largamente dalle e nelle istituzioni politiche, amministrative, economiche» ${ }^{68}$.

\section{All' insegna dello stile e dell' affabilità}

Nel volgere di qualche anno il quadro italiano cambia e con esso il corredo dei politici ${ }^{69}$. Tra la fine degli anni Cinquanta e lungo il successivo decennio, con il trionfo del Life American Style, l' «impero irresistibile» - per dirla con Victoria De Grazia - dei consumi si insinua prepotentemente nella vita delle e degli italiani inducendo ad altri bisogni ed eleggendo le donne

64. E. Scarpellini, "Gli studi sulla moda come settore storiografico emergente", Memoria e ricerca, 50, 2015, 11-25, la citazione è a p. 20. Si rimanda a B. Burman, C. Turbin (Dir.), Material strategies. Dress and gender in historical perspective, Malden-Mass, 2003.

65. D. J. Cole, N. Deihl (Dir.), Storia della moda dal 1860 ad oggi, Torino, 2016, 69. Per i colori si veda almeno M. Pastoreau, Bleu. Histoire d'une couleur, Paris, 2000. M. Pastoureau, Les couleurs de notre temps, Paris, 2003.

66. E. Mora, "La moda italiana e l'uomo integrato", en E. dell'Agnese, E. Ruspini, (Dir.), “Mascolinità...”, op. cit., 103-126, la citazione è a p. 105.

67. E. Mora, “La moda italiana..., op. cit., 106-108.

68. E. Mora, "La moda italiana..., op. cit., 107-108

69. Si veda S. Gundle, I comunisti italiani tra Hollywood e Mosca. La sfida della cultura di massa (19431991), Firenze, 1995. 
a consumatrici di merci destinate alla cura e alla valorizzazione del corpo ${ }^{70}$. Il discorso sulla moda ne è investito e mostra presto più di un'ambivalenza, lo conferma il cinema cartina al tornasole dei processi sociali. Se Luciano Emmer, in Le ragazze di Piazza di Spagna, del 1953, ambientato nella sartoria delle sorelle Fontana, vera e propria eccellenza in quegli anni, fa della moda il «sinonimo di perdizione», al contrario - osserva Silvia Gnoli - in Il mondo le condanna, di Franciolini, l'ex prostituta poi indossatrice Renata vi trova la via della salvezza ${ }^{71}$. La moda assume sensi diversi e, con il tempo, la demonizzazione va affievolendosi. La società dei consumi giudica «la politica dell'apparire» in rapporto ai suoi canoni estetici: i politici sanno seguire la moda? Sono adeguati? Queste le domande di fondo sottese a tanti servizi. Lungo gli anni Sessanta, la rappresentazione di uomini e donne cambia sensibilmente, non si richiama alla civetteria o alla sciatteria, si diffondono ritratti di deputate in abiti da cerimonia, da sera, nell'onnipresente tailleur. Ancora una volta, insieme agli scenari che fanno da cornice, è l'abito a tracciare il confine tra il formale e il privato e si afferma come segnale dell'adesione al rituale del vestire che muta con il cambiare dell'ora e del luogo ${ }^{72}$. Compaiono abiti leggeri e informali indossati durante le vacanze e il tempo libero. Non è tanto diverso per gli uomini politici che nel dopoguerra vediamo in tenuta da montagna, sono note a riguardo le fotografie di De Gasperi e Togliatti ${ }^{73}$. Qualche anno dopo, quando il mare diventa sinonimo di vacanza e di benessere, i politici appaiono sotto gli ombrelloni nelle spiagge, in barca, ma anche fasciati in eleganti abiti completi scuri, con tanto di papillon: la moda oramai non è più ostentazione di lusso e di ricchezza ${ }^{74}$ ma segno di adeguamento ai tempi e modernità, il look resta fondamentale nella sessuazione sociale dei corpi ${ }^{75}$. La moda suggerisce nuovi tagli, uno stile più agile e disinvolto e certamente la palette si allarga ad altre tinte e nuances: motivi stampati geometrici o a fiori occupano pure gli armadi delle elette come testimonia una bella fotografia di Giglia Tedesco, del Pci, mentre il pratico e lineare chemisier trionfa adottato da coloro che propendono per uno stile sobrio e ricercato, come conferma un' immagine di Tullia Carrettoni del 1963 che esibisce un abito chiaro con tasche alte, una cintura a sottolineare il punto vita, collana di perle, orecchini, perfetta acconciatura corta e unghie laccate ${ }^{76}$.

70. S. Gundle, “I comunisti italiani..., op. cit.

71. S. Gnoli (Dir.), Moda italiana 1950-1970, s.1., 2001.

72. A. C. Quintavalle, “Archivio...”, op. cit., XI: «La moda portata è una scrittura, sarebbe a dire che è una scrittura del corpo, e quindi scena, rappresentazione, invenzione di una gestualità individuale».

73. Si veda M. Marsili, "De Gasperi and Togliatti: political leadership and personality cults in post-war Italy”, Modern Italy, 3, 1998, 249-261.

74. S. Gnoli, "Un secolo..., op. cit., 178. Per un quadro sul tema moda e costruzioni di genere si veda D. Crane, Questioni di Moda. Classe, genere e identità nell'abbigliamento, Milano, 2004. S. Cassagnes-Brouquet, Ch. Dousset-Seiden, "Genre, normes et langages du costume", Clio. Femmes, genre, Histoire. Costumes, 36, 2012, 7-19; Clio. Femmes, genre, Histoire. Objes et fabrication du genre, 40, 2014.

75. C. Guillaumin, “Il corpo..., op. cit., 307-340.

76. htpp://www.eletteedeletti.it; Stefano Pivato ha efficacemente descritto questi cambiamenti in riferimento alla parabola ascendente di Valentina Tereschkova, l'astronauta sovietica, nel 1963 prima donna nello spazio, esaltata anche per le sue virtù fisiche. Un personaggio che rappresenta un paradigma della evo- 
Sempre negli anni Sessanta si diffonde l'uso degli accessori, segno e strumento per eccellenza della differenziazione ${ }^{77}$ :

Cosmetics, fashion goods, accessories, and other goods that could be worn or carried fuelled the immagination. They were especially suggestive - scrive Stephen Gundle - since they bore directly on personal identity and promised the immediate realization of a trasformation of the self into something different and better ${ }^{78}$.

Storicamente limitati per gli uomini, gli accessori si confermano come proiezione della femminilità e - una volta usciti dalle strettoie imposte dalla Ricostruzione ai consumi - saranno largamente usati nella definizione della donna e dell' uomo politico ${ }^{79}$. Foulard, spille, fili di perle, orecchini, borse per le signore; occhiali, in alcuni casi da sole per gli uomini, come un tempo cappelli e bastoni, fanno la loro comparsa.

I bastoni che avevano troneggiato in tanti ritratti tra Otto e Novecento, sono scomparsi, unico scenario che li contempla è quello alpino, lo stringe tra le mani anche Nilde Iotti. Si indossano occhiali con montature lineari e semplici, l'orologio è portato con discrezione, all'occhiello della giacca raramente si vedono fiori, fanno eccezione le serate di gala, mentre dal taschino si affacciano lindi e stiratissimi, probabilmente inamidati, fazzoletti bianchi: sono segno di distinzione ma il loro candore rimanda all' igiene, compresa quella morale, e al decoro ${ }^{80}$. Le cartelle in cuoio o pelle divengono una sorta di protesi dall' uomo politico, al loro interno si raccolgono ordinatamente carte e appunti di lavoro: sono l'emblema della laboriosità.

La tavolozza del politico vede l'aggiunta di nuovi elementi, colori chiari in estate, paltò in tessuto jacquard o occhio di pernice, abiti in principe di Galles, qualcuno - è il caso di Giulio Andreotti - lasciate le scarpe con i lacci, indossa pratici, robusti mocassini. Le cravatte passano dalla tinta unita alla fantasia rigata, qualcuno azzarda decisi contrasti cromatici.

I politici appaiono disinvolti, il cappello non è più segno di distinzione né per i signori né per le signore. Ne indossa con disinvoltura uno a larghe falde De Gasperi; Togliatti a capo scoperto sfoggia un taglio di capelli che lascia libera la fronte; Nenni indossa il basco che può essere letto come segno di evasione e di differenziazione dal contesto. A colpire sono le

luzione che investe la raffigurazione del "corpo della comunista". S. Pivato, I comunisti sulla luna. L'ultimo mito della Rivoluzione russa, Bologna, 2017.

77. R. Barthes, L'Overture sociologique, Paris, 1985, sostiene che gli accessori sono costituiti da differenze.

78. S. Gundle, Glamour. A History, London, 2008, 389.

79. Sull' uso degli accessori per marcare le differenze individuali e identitarie e la sua evoluzione si veda A. Giancola, "Accessori: strategie della moda e identità giovanile", en B. Valli, B. Barzini, P. Calefato (Dir.), Discipline della moda. L'etica dell'apparenza, Napoli, 2003, 237-250; L. Schettini, "Oggetti del desiderio o corpi del reato? Abiti, accessori, fotografie e modelli di genere tra Otto e Novecento", La camera blu. Rivista di studi di genere, 9, 2013, e della stessa Il gioco delle parti. Travestimenti e paure sociali tra Otto e Novecento, Firenze, 2012.

80. Sulla storia degli oggetti e gli studi sulla cultura materiale si veda C. Lunghi, M. A. Trasforini (Dir.), La precarietà degli oggetti: estetica e povertà, Roma, 2010. 
pose, comprese quelle dei ritratti. Le parlamentari, come i loro colleghi, acquistano nel tempo corporeità, distribuiscono sorrisi che infondono simpatia e serenità. La «politica del sorriso» suggerita da Concetto Marchesi al PCI affascina tutte le forze politiche ${ }^{81} \mathrm{e} \mathrm{la} \mathrm{figura} \mathrm{del} \mathrm{leader}$ vi si adegua smorzando l'atteggiamento distaccato del politico di età liberale e prendendo nettamente le distanze dalle pose virili di Mussolini ${ }^{82}$. Si assiste alla pubblicazione di immagini meno statiche che riducono la distanza tra il deputato e l'elettorato.

Nel dopoguerra il «privato» dell' uomo politico resta a lungo un ibrido: il giardino della propria casa, lo sfondo di panorami alpini, mentre la dimensione relazionale è citata con lo scopo di rafforzare la completezza del soggetto che resta saldamente collocato nello spazio pubblico $^{83}$. Ritratti nella propria abitazione, nel proprio giardino, al caminetto, essi godono il meritato riposo tra la moglie e i figli. Si tratta però di brevi pause, la mente del politico resta rivolta alla gestione della cosa pubblica anche quando viene colto in un momento conviviale, come al tavolo di un ristorante, o nel proprio salotto. Gli eletti appaiono stereotipicamente caratterizzati da virtù pubbliche ed abitano lo spazio pubblico, il loro corpi sono proiettati verso l'esterno caratterizzati dall'azione. La paternità, elemento tutt'altro che trascurabile nella definizione del maschile, ha subito un duro colpo con la guerra, tanto da poter richiamare ad un' intera generazione di orfani, senza padri reali perché partiti in guerra né simbolici data la catastrofe cui le precedenti generazioni hanno condotto l'Italia ${ }^{84}$. Le fotografie presentano i politici con figlie e mogli, esse sono una sorta di cornice che ingentilisce il quadro. Nel caso di Togliatti, l'enfasi sulla figlia adottiva conferma ideali e appartenenza politica. Le immagini che riguardano gli interni privilegiano la scrivania oggetto simbolo del lavoro intellettuale che il ruolo di eletto richiede: il privato della dimensione maschile sta cambiando, ma continua a restare escluso o, comunque, sullo sfondo del palcoscenico pubblico. Almeno fino al Cinquanta, per le elette, invece, in risposta all' immagine radicata della ribelle sguaiata e pericolosa, capace di soggiogare gli uomini, partiti di massa e associazioni femminili scelgono una strategia difensiva che trova espressione nella maternità, quasi a dimostrare la possibilità di coniugare ruoli considerati antitetici, essere madre e professionista della politica al contempo, e di scongiurare l'allarme sulla mascolinizzazione. Si riproduce così quella costruzione che affida agli uomini la padronanza dello spazio e la proiezione del corpo verso l'esterno,

81. Si vedano S. Gundle, “I comunisti italiani...", op. cit.; F. d’Almeida, "Vendere al meglio la propria immagine: mimica facciale e seduzione da parte dei parlamentari francesi e italiani”, en A. Baravelli (Dir.), Propagande contro. Modelli di comunicazione politica nel XX secolo, Roma, 2005, 227-246. Su consumi e genere resta fondamentale a V. De Grazia, E. Furlought (Dir.) The Sex of Things: Gender and Consumption in Historical Perspective, Berkeley, 1996.

82. S. Gundle, From Mussolini to Berlusconi: Masculinity and Political and Leadership in Post-war Italy, London, 2018.

83. Sul rapporto spazi-genere e sulla città costruzione discorsiva, M. A. Trasforini, Arte a parte. Donne artiste tra margini e centro, Milano, 2000; con particolare riferimento al saggio di Pollock e della stessa "Le flâneuses...”, op. cit.; G. Nuvolati, L'interpretazione dei luoghi: flânerie come esperienza di vita, Firenze, 2013. Ch. Bard (Dir.), Le genre des territoires: féminin, masculin, neutre, Angers, 2004.

84. A. Gibelli, Il popolo bambino. Infanzia e nazione dalla Grande guerra a Salò, Torino, 2005. 
alle donne il ripiegamento sul proprio spazio corporeo. La limitazione spaziale del genere femminile ha quale risultato una pratica del corpo orientata verso ambiti ridotti quali la casa, la famiglia. I corpi femminili, inscritti in una spazialità statica, rimandano alla immobilità, mentre cornice familiare e relazionale, si è accennato, fanno da sfondo alle immagini delle elette, sebbene le loro biografie rivelino un livello di esperienze politiche significative, esse continuano almeno fino alle soglie degli anni Cinquanta a essere valorizzate in quanto madri.

Successivamente, i processi di modernizzazione che vedono affacciarsi, seppure a fatica, altri modelli e stili di vita, oltre ad una visibilità femminile nel mondo del lavoro e del terziario, investono pure la rappresentazione della politica. Si coglie un aumento quantitativo di immagini che ritraggono i politici in casa, in vacanza, in occasioni conviviali. Tale orientamento interviene sull' immagine austera, sull" "uomo tutto d' un pezzo", per rappresentare soggetti in carne ed ossa con interessi e sentimenti non troppo distanti da quelli nutriti dall'elettorato, mentre le pose disinvolte rimandano alla relazione e all'affabilità. Se nel 1954 Amintore Fanfani è ripreso con la madre che sembra porsi garante dell'eleganza del figlio, nel decennio successivo sul divano con la moglie e il figlio è fotografato Loris Fortuna; Cesare Merzagora è, invece, ripreso a passeggio con la moglie: le pose sono assai meno convenzionali rispetto al passato e sembrano suggerire un nuovo «posizionamento» delle donne nella famiglia, non più ancelle - versione dominante il primo decennio della Repubblica - ma come compagne di vita.

Il «privato» pridilige diversi palcoscenici: le vacanze, gli interessi culturali e gli svaghi, i viaggi. La narrazione è più dinamica e marginalizza l'icona della perfetta casalinga. Alle soglie degli anni Cinquanta le rappresentazioni diffusissime nel dopoguerra, dell' uomo soggiogato dalle virago appartengono oramai al passato. L'immagine di soggetti sopraffatti, poco virili è utilizzata sostanzialmente per indicare rapporti gerarchici tra i diversi partiti. La perdita di virilità resta centrale nella rappresentazione dei «deboli» ma diviene metafora dei rapporti politici interni ai parlamentari. Senza lesinare si ricorre al binomio oppositivo mascolinità-potere, femminilità-sottomissione, sottintesi restano i canoni egemonici eterosessuali. La messa in ridicolo - se si escludono i trinariciuti e le trimammellute di Giovanni Guareschi - passa attraverso categorie e valutazioni inscritte nella dimensione politica: vincitori-vinti, forti-deboli, astuti-ingenui, arguti-ignoranti ${ }^{85}$.

La «costruzione dei corpi» della donna e dell' uomo politico, il loro «posizionamento» nell'arena pubblica possono concorrere alla riproduzione di disuguaglianze e asimmetrie e possono contribuire alla lettura delle culture politiche dell' Italia Repubblicana.

Potrebbe essere utile sondare in profondità queste rappresentazioni allargando la visuale ai media che, negli anni Sessanta, acquistano centralità nella formazione di nuovi immaginari ${ }^{86}$.

85. M. Y. Ostrogorski, Democrazia e partiti politici, a cura di G. Quagliarello, Milano, 1991.

86. M. Ravveduto, La nazione del miracolo. L'Italia e gli italiani tra Storia, memoria e immaginario (19631964), Roma, 2018. 